\title{
Bipartite Adenoviral Vector encoding hHGF and hIL-1Ra for Improved Human Islet Transplantation
}

\author{
Ravikiran Panakanti and Ram I. Mahato* \\ Department of Pharmaceutical Sciences, University of Tennessee Health Science Center, \\ Memphis, TN, USA
}

\begin{abstract}
Purpose-Ex vivo gene therapy can improve the outcome of islet transplantation for treating type I diabetes. Hepatocyte growth factor (HGF) increases $\beta$-cell proliferation and promotes revascularization of islets, while interleukin-1 receptor antagonist (hIL-1Ra) inhibits islet cell apoptosis.

Methods-We constructed Adv-hHGF-hIL-1Ra by cloning hHGF and hIL-1Ra coding sequences and polyA signal under separate CMV promoters in Adenoquick plasmid.

Results-There was dose and time dependent expression of these genes after transduction of AdvhHGF-hIL-1Ra into human islets. Compared to un-transduced islets, hHGF and hIL-1Ra gene expression at protein levels was more than 60 and 40 times higher at $1000 \mathrm{MOI}$, respectively. Transduced islets were viable after incubation with the cocktail of TNF- $\alpha$, IL- $1 \beta$ and IFN- $\gamma$, as evidenced by insulin release in response to glucose concentration. Co-expression of hHGF and hIL-1Ra led to significant decrease in caspase- 3 induced by the cytokines. Compared to untransduced islets, transduction of islets with Adv-hHGF-hIL-1Ra at $1000 \mathrm{MOI}$ prior to transplantation under the kidney capsules of streptozotocin-induced-diabetic NOD-SCID mice reduced blood glucose levels, and increased serum insulin and c-peptide levels.
\end{abstract}

Conclusions-Transduction of islets with Adv-hHGF-hIL-1Ra efficiently expresses both growth factor and antiapoptotic genes, decreases caspase- 3 and improves the outcome of islet transplantation.

\section{Keywords}

Human islets; adenoviral vectors; hepatocyte growth factor; hIL-1Ra; islet transplantation

\section{Introduction}

Type 1 diabetes is a chronic inflammatory autoimmune disease resulting from the loss of islet function, requiring life-long insulin therapy. Human islet transplantation has the potential to replace pancreatic endocrine function in patients with type 1 diabetes $(1,2)$. One major drawback to this approach is the requirement of islets derived from two to four cadaver donors to treat a single patient with diabetes. In addition, required islet mass has been seen in successfully transplanted recipients compared with healthy people (3). Thus, an enormous shortage of human islets is a barrier to the use of islet transplantation on a larger scale. Despite recent success, islet transplantation still lags behind primarily because a large number of transplanted islets often do not function (4).

*Corresponding Author: Ram I Mahato, PhD, Department of Pharmaceutical Sciences, University of Tennessee Health Science Center, 19 S Manassas, RM 224, Memphis, TN 38103-3308, USA, Tel: 901-448-6929; Fax: 901-448-2099, rmahato@utmem.edu, http://cop.utmem.edu/rmahato. 
Islets have an intricate microvasculature, which is disrupted during islet isolation and purification process. Therefore, revascularization to the transplanted islets is essential for delivery of nutrients and oxygen to the inner core of islets for their survival (5-8). Hepatocyte growth factor (HGF) is a potent mitogen for human islets and is known to increase the proliferation and expansion of $\beta$-cells and promotes revascularization of islets, while maintaining physiologic glucose responsiveness after transplantation (9). Overexpression of HGF in the $\beta$-cells of transgenic mice directed by the rat insulin type II promoter (RIP) has shown to increase $\beta$-cell mass and proliferation, and improve islet function and islet transplant outcome (10). Ex vivo transduction of islets with an adenoviral (Adv) vector encoding HGF has been reported to improve the outcome of islet transplantation (11). Intravenous injection of Adv-HGF in streptozoticin (STZ)-induced diabetic mice has shown to ameliorate hyperglycemia and prolong survival period in the diabetic mice (12). Moreover, HGF gene transfer has also shown to increase the expression of three key $\beta$-cell genes, glucokinase, Glut- 2 (the $\beta$-cell glucose transporter), and insulin (13).

Success of islet transplantation not only depends on islet revascularization, but also on its protection from apoptosis induced by hypoxia, oxidative stress and inflammatory cytokines released from resident macrophages and infiltrating immune cells at the transplantation site (14). More than 70\% of islets become inactive in 7-14 days post-transplantation (15). Among these cytokines, IL-1 $\beta$ plays a key role in apoptotic cell death. Inhibition of IL- $1 \beta$ receptor binding using interleukin-1 receptor antagonist (IL-1Ra) resulted in improved islet survival and function (16-18). HGF has also been reported to induce anti-apoptosis (19).

Considering the favorable uses of hHGF and hIL-1Ra, in this study we constructed a bipartite Adv vector encoding these two genes (Adv-hHGF-hIL-1Ra) by cloning hIL-1Ra and hHGF under a separate cytomegalovirus (CMV) promoter. We determined transduction efficiency, islet function, and caspase-3 release upon incubation with inflammatory cytokine cocktail of TNF- $\alpha$, IL- $1 \beta$ and IFN- $\gamma$. We then transplanted islets under the kidney capsules of nonobese diabetic/severe combined immunodeficient (NOD-SCID) mice after transduction with AdvhHGF-hIL-1Ra and determined the levels of blood glucose and serum insulin. Finally, the islet bearing kidney sections of these diabetic mice were stained for human insulin, hHGF and von Willebrand factor (vWH).

\section{Materials and Methods}

\section{Materials}

Agarose, tris-borate-EDTA buffer, goat serum, glucose and haematoxylin solution were purchased from Sigma-Aldrich Co. (St. Louis, MO). Fetal bovine serum (FBS) and CMRL-1066 media were purchased from MediaTech Cellgros (Herndon, VA). Phosphate buffer solution (PBS) was purchased from GIBCO-BRL (Gaithersburg, MD). Human HGF and IL-1Ra ELISA kits and cytokines IL- $1 \beta$, TNF- $\alpha$ and IFN- $\gamma$ were purchased from R\&D Systems (Minneapolis, MN). Human Insulin ELISA kits were purchased from Alpco Diagnostics (Windham, NH). RNA extraction kit and Caspase-Glo 3/7 assay kit were procured from Promega (Madison, WI). SYBR Green real-time PCR master mix and reverse transcription reagents were purchases from Applied Biosystems (Foster city, CA). Sodium citrate was procured from Curtin Matheson Science Inc. (Houston, TX.

\section{Methods}

Construction of Adv-hHGF-hIL-1Ra-A bipartite Adv vector expressing hHGF and hIL-1Ra genes was constructed with the help of OD 260, Inc. (Boise, ID). Briefly, the hHGF cDNA was excised from pCMV-hHGF using BamHI, and cloned into the BamHI site of pE1.2, which contains a CMV promoter, multiple cloning site, and bGH polyA signal. Similarly, 
hIL-1Ra cDNA was excised from pUMVC-hIL-1Ra vector using ScaI, separated by gel fractionation, and purified using Promega PCR and cleanup kit (Promega, Madison, WI). The hIL-1Ra fragment along with rabbit $\beta$-globin polyA was then cloned into $\mathrm{pE} 3.1$ shuttle plasmid using NotI restriction site. The $\mathrm{pE}$ 3.1 IL-1Ra plasmid was further modified by removing the intron adjoining CMV promoter. All the ligation reactions were done at 1:3 and 1:10 ratios using T4 DNA ligase and then transformed in super competent TOP 10 cells and amplified in terrific broth media. The plasmids were then purified using Gen Elute plasmid Mini prep kit (Sigma-Aldrich, St. Louis, MO). The plasmids pE 1.2-hHGF and pE 3.1-hIL-1Ra were digested with AlwNI and DraIII respectively and the excised fragments were purified on agarose gel. These fragments were ligated with SfiI-digested AdenoQuick 13.1 plasmid simultaneously using T4 DNA ligase overnight at $16^{\circ} \mathrm{C}$. The ligated products were packaged into phage lambda particles using MaxPlax packaging extract (Epicentre, Madison, WI). The packaging mixture was used to infect E. coli XL-1Blue (Stratagene) and clones were selected on LB supplemented with $25 \mu \mathrm{g} / \mathrm{ml}$ kanamycin and $50 \mu \mathrm{g} / \mathrm{ml}$ ampicillin. The Pac I linearized cosmid was transfected into 293 cells to rescue the virus. Three viral plaques were amplified, and their genomic DNAs were extracted. The presence of hHGF and hIL-1Ra expression cassettes in these three vectors was confirmed by restriction analysis with SpeI and EcoRV, respectively. The clone containing the two genes was infected to 293 cells. Both the cells and the medium were harvested and frozen/thawed three times. This viral extract was further infected into 293 cells, amplified and purified on two $\mathrm{CsCl}$ gradients. The bands harvested were dialyzed against $3 \times 11$ GTS buffer $(25 \%$ glycerol, $25 \mathrm{mM} \mathrm{NaCl}, 20 \mathrm{mM}$ Tris- $\mathrm{HCl}(\mathrm{pH} 8)$ for 12 hours at $4{ }^{\circ} \mathrm{C}$. The dialyzed viral suspension was collected, filtered and stored at $-70^{\circ} \mathrm{C}$.

Determination of Viral Titer-The infectious viral titer was also determined by using an Adeno-X rapid titer kit from Clontech (Mountain View, CA). Different dilutions of Adv-HGFhIL-1Ra was taken and transduced to AD-293 cells, using un-transduced cells as a control. After $48 \mathrm{~h}$ post-transduction, AD-293 cells were fixed and incubated with Anti-Hexon antibody, followed by a secondary horse radish peroxidase (HRP)-conjugated antibody. Then the cells were stained with $3,3^{\prime}$ diaminobenzidine tetrahydrochloride (DAB) substrate and positively stained cells were counted under a microscope, and the titer was determined.

Islet Culture and Transduction-Human Islets were received from one of the several Islet Cell Resource (ICR) Centers in culture media at $4^{\circ} \mathrm{C}$. On receiving, the islets were cultured in CMRL-1066 medium containing $10 \%$ fetal bovine serum (FBS) and $1 \%$ Penicillin

Streptomycin. Islets were then transduced with Adv hHGF-hIL-1Ra, while non-transduced islets were used as controls. To determine the optimal multiplicity of Infection (MOI) of Adv in terms of plaque forming units (pfu) per islet equivalent, 1000 islets were incubated with diluted adenovirus at 100, 500,1000, 2000 and $5000 \mathrm{MOI}$ in 300 $\mu \mathrm{l}$ media in a 96 well plate for $12 \mathrm{~h}$, followed by washing and further culture in $1 \mathrm{ml}$ of media in 24-well plates. Gene expression was determined at days 1 and 3 by quantifying secreted protein in the media by ELISA (R\& D Systems, Minneapolis, MN). Total protein of the islet cell extracts was also determined using the bicinchonic acid (BCA) protein assay kit to normalize the results. Three samples per group were included for the experiment.

Quantitative Real-time PCR-To determine hHGF and hIL-1Ra gene expression in human islets at the mRNA level by real time RT-PCR, at day 3 post-transduction of islets with AdvhHGF-hIL-1Ra the islet pellet was collected for isolation of total RNA using RNAeasy mini isolation kit from Promega. RNA concentration was measured by UV spectrophotometry using Biomate 3 spectrophotometer. Two hundred and fifty nanograms (ng) of extracted RNA was converted into cDNA using MultiScribe reverse transcriptase and random hexamers (Applied Biosystems, Inc., Foster City, CA) by incubation at $25^{\circ} \mathrm{C}$ for $10 \mathrm{~min}$, followed by reverse transcription at $48^{\circ} \mathrm{C}$ for $30 \mathrm{~min}$ and enzyme inactivation at $95^{\circ} \mathrm{C}$ for $5 \mathrm{~min}$. In all $3.85 \mu \mathrm{l}$ of 
cDNA was amplified by real-time RT-PCR. To determine the level of HGF expression, the following hHGF-specific primers were used: Forward: 5'- CCT ATG CAG AGG GAC AAA GGA A - 3'; Reverse: 5'- GCC TTG CAA GTG AAT GGA AGT CCT - 3' (NCBI Accession\# 291674). In case of hIL-1Ra both, forward (5'-ATC CAG CAA GAT GCA AGC CCT-3') and reverse (5'-TTC GTC AGG CAT ATT GGT GAG GCT-3') primers were designed from the gene sequence, (NCBI Accession \# AK290898). The PCR conditions included denaturation at $95^{\circ} \mathrm{C}$ for $10 \mathrm{~min}$, followed by 40 cycles of amplification by sequential denaturation at $95^{\circ} \mathrm{C}$ for $15 \mathrm{sec}$ and primer annealing as well as strand extension for $1 \mathrm{~min}$. hHGF and hIL-1Ra gene expression was normalized to 19s rRNA as internal control.

To confirm the amplification specificity, the PCR products were subjected to melting curve analysis. The expressed HGF and hIL-1Ra levels were quantified and normalized to the total amount of cDNA used. Threshold cycle number was compared between Adv-hHGF-hIL-1Ra with the same parameters using non-transfected islets as controls.

In Vitro Islet Function after Infection-The in vitro function of human islets after infection with Adv-hHGF-hIL-1Ra was determined by the static incubation method. Noninfected islets were used as control. Transfected media was removed by centrifugation at $300 \mathrm{~g}$ and the islets were sequentially incubated at $37^{\circ} \mathrm{C}$ for $1 \mathrm{hr}$ in the media containing $2.5 \mathrm{mM}$ (basal) and $22 \mathrm{mM}$ glucose (stimulated). After incubation, supernatants were collected and analyzed for insulin release by ELISA (Alpco Diagnostics). Insulin secretion was expressed as $\mu \mathrm{U} / \mathrm{ml}$ and the ratio of insulin levels at $22 \mathrm{mM}$ to $2.5 \mathrm{mM}$ glucose was used to calculate the stimulation index.

Cytoprotective effect of Adv-hHGF-IL-1Ra-One thousand islets per well of 96 well plates were infected with 1000 MOI of Adv-HGF-hIL-1Ra at 500, 1000 and 2000 MOIs. At $12 \mathrm{~h}$ post-transduction, islets were washed and incubated with a cocktail of IL- $1 \beta(10 \mathrm{ng} / \mathrm{ml})$, TNF- $\alpha(10 \mathrm{ng} / \mathrm{ml})$ and IFN- $\gamma(50 \mathrm{ng} / \mathrm{ml})$ cytokines. Non-transduced islets with and without cytokine treatment were used as controls. Blank media was also used the negative control. At day 3 post transduction islets were collected from each well and were lysed with lysis buffer to measure the caspase- 3 concentration using the Caspase-Glo 3/7 Assay kit (Madison, WI).

Western Blotting-At day 3 post-transduction of human islets with Adv-hHGF-hIL-1Ra, protein extracts were prepared by washing cells with PBS and lysing in RIPA buffer containing protease inhibitor (Roche, Basel, Switzerland). Human islets were homogenized and lysates were spun at $15000 \mathrm{~g}$ for $15 \mathrm{~min}$ to remove insoluble material. Protein concentrations were determined by BCA protein assay. Proteins were resolved on 4-15\% sodium dodecyl sulfate polyacrylamide gel electrophoresis (SDS-PAGE) (Bio-Rad, Hercules, CA) and transferred to a PVDP membrane (Bio-Rad, Hercules, CA), blocked with TBST buffer composed of $50 \mathrm{mM}$ Tris (pH 7.6), $150 \mathrm{mM} \mathrm{NaCl}$ and $0.05 \%$ Tween supplemented with $5 \%$ fat-free milk at $4{ }^{\circ} \mathrm{C}$ for $1 \mathrm{~h}$. After a brief rinse, the membrane was incubated overnight at $4^{\circ} \mathrm{C}$ in TBST containing $0.5 \%$ milk with mouse anti-human Bcl-2 (Santacruz, CA) (1:200) and mouse anti-human Bax (Santacruz, CA). Goat anti-human Actin monoclonal antibody (Santacruz, CA) (1:2000) was used for detecting an internal control protein. Then, blots was washed four times using TBST for $1 \mathrm{~h}$ at room temperature. Immunodetection was performed using a horseradish peroxidase (HRP) CHEMI Blot Starter Kit (Bio-rad, Hercules, CA) according to the manufacture's instruction.

Islet transplantation studies-Animal experiments were performed as per the NIH (http://grants1.nih.gov/grants/olaw/references/phspol.htm) and institutional animal care and use guidelines using the approved protocol. To induce diabetic animal model, streptozotocin (STZ) $(40 \mathrm{mg} / \mathrm{kg})$ was administered to NOD-SCID mice by intraperitoneal injection for 5 consecutive days. Animals were considered to be diabetic after two consecutive measurements 
of blood glucose $\geq 300 \mathrm{mg} / \mathrm{dl}$ using a One Touch Ultra Glucometer (LifeScan, Inc.). Before transplantation, human islets were transduced by Adv-hHGF-hIL-1Ra at the dose of $1000 \mathrm{MOI}$ for $12 \mathrm{~h}$ and washed with PBS. About 1000 transduced or non-treated islets were transplanted under the left kidney capsules of diabetic mice. The nonfasted glucose levels were measured from the snipped tail of each animal up to 28 days post-transplantation. At day 28 posttransplantation, the mice were then anesthetized to collect blood to measure serum insulin and c-peptide levels by ELISA (Alpco Diagnostics, Salem, NH). At day 28, the graft-bearing kidneys were removed from some animals to confirm the function of islet grafts by the return of blood glucose levels to $\geq 300 \mathrm{mg} / \mathrm{dL}$ for two consecutive days.

Intraperitoneal Glucose Tolerance Test-Twenty seven days after islet transplantation under the kidney capsules of STZ-induced NOD-SCID diabetic mice, glucose tolerance was analyzed in overnight fasted mice by intraperitoneal injection of $2 \mathrm{~g}$ of glucose $/ \mathrm{kg}$ of body weight as described by Garcia-Ocana et al.(11) Blood samples were obtained from the snipped tail and analyzed for glucose levels using a One Touch Ultra Glucometer (LifeScan, Inc.).

Immunohistochemistry and morphometric analysis-To determine hHGF expression and angiogenesis, transplanted mice were sacrificed, kidneys were isolated, washed with PBS, fixed in $4 \%$ formaldehyde overnight, and embedded in paraffin as described before (6). Sections of 5-7 $\mu \mathrm{m}$ thickness were cut and immunostained with rabbit anti-insulin, hHGF and hvW primary antibodies, respectively. The immunoreactivity was detected using goat antirabbit IgG, $\mathrm{H} \& \mathrm{~L}$ chain specific peroxidase conjugate and subsequent incubation with DAB substrate. For morphometric analysis, hHGF and hvWF-positively immunostained color (brown in each case) was selected for quantification of the relative intensity using NIH Image 1.62 software to determine the mean values, which were subsequently compared between AdvhHGF-hIL-1Ra transduced and control islet transplanted diabetic recipient mice.

Statistical Analysis-Statistical significance of the difference between the two groups was determined by unpaired t-test and between several groups by one-way ANOVA.

\section{Results}

\section{Construction of Adv-hHGF-hIL-1Ra}

Adv-hHGF-hIL-1Ra is a serotype 5 based replication deficient Adv vector that contains a CMV promoter, hHGF cDNA and rabbit $\beta$-globin poly $\mathrm{A}$ in the $\mathrm{E}-1$ region and a CMV promoter, hIL-1Ra cDNA and rabbit $\beta$-globin poly A in the E-3 region (Fig. 1). hHGF and hIL-1Ra coding sequences and poly A signals were placed under the control of a separate CMV promoter cloned into $\mathrm{pE} 1.2$ and $\mathrm{pE} 3.1$. The resulting vectors were combined in Adenoquick 13.1 to generate a cosmid that contains the entire sequence of recombinant adenovirus. The presence of hHGF and hIL-1Ra expression cassettes in these vectors was confirmed by restriction digestion with Hind III (data not shown). The number of viral particles in the stock solution of Adv-hHGF-hIL-1Ra was determined using an Adeno $\times$ rapid titer kit from Clontech (Mountain View, CA) and the titer was calculated to be $1.24 \times 10^{\wedge} 9 \mathrm{ifu} / \mathrm{ml}$ (infectious units/ $\mathrm{ml})$.

\section{Transduction Efficiency of Adv-hHGF-hIL-1Ra}

Following the transduction of human islets with Adv-hHGF-hIL-1Ra, the level and duration of hHGF and hIL-1Ra proteins increased with increase in MOI. hHGF and hIL-1Ra protein levels at day 3 post-transduction was over 60 and 40 times higher than that of the untransduced islets, respectively (Fig. 2), indicating that Adv-hHGF-hIL-1Ra transduction would lead to high local concentrations of hHGF and hIL-1Ra in the immediate vicinity of islets post transplantation. We also determined hHGF and hIL-1Ra gene expression at mRNA levels by 
real time RT-PCR. As shown in Fig. 3, the normalized mRNA concentration to that of the control was significantly increased with increase in MOI of Adv-hHGF-hIL-1Ra. Gene expression at mRNA level of hHGF and hIL-1Ra was more than 60 and 40 times higher than that of untransduced islets, respectively (Fig. 3). Each graph is indicative of the relative increase in expression of hHGF and hIL-1Ra independently. This shows that Adv-hHGF-hIL-1Ra can simultaneously express both genes with high efficiency. The mRNA concentrations of each gene were normalized to that of the control i.e. untransduced islets. The amplification specificity was confirmed by melting peak analysis, which showed a single predominant peak each for s19 $\left(84.5^{\circ} \mathrm{C}\right)$, hHGF $\left(78.8^{\circ} \mathrm{C}\right)$ and hIL-1Ra $\left(85.5^{\circ} \mathrm{C}\right)$, respectively (data not shown).

\section{Effect of Viral Transduction on Islet Function}

To determine whether insulin secretion was adversely affected by transduction of islets with Adv-hHGF-hIL-1Ra, insulin secretion in response to glucose challenge was carried out at day 3 post-transduction at 500, 1000 and 2000 MOI, while non-transduced islets were used as the control. Glucose challenge was determined by quantifying glucose stimulated insulin release at basal level $(2.5 \mathrm{mM})$ and stimulated $(22 \mathrm{mM})$ glucose concentrations. There is a significant decrease in the stimulation index of un-transduced control islets when incubated with the cytokine cocktail compared to that in the absence of cytokine incubation (Fig. 4). Stimulation indices of Adv-hHGF-hIL-1Ra-transduced islets were almost identical to that of untransduced islets, when they were not incubated with the cytokine cocktail, suggesting that the transduction process does not cause any harm to the islets. Although incubation with the cytokine cocktail also led to decrease in the stimulation indices of Adv-hHGF-hIL-1Ra transduced islets, this decrease was much less compared to that of the un-transduced control islets, possibly due to the protective effect of hHGF and hIL-1Ra co-expression (Fig. 4).

\section{Apoptosis of Islets}

Apoptosis of islets leads to their impaired function and subsequent death, and thus it is an important detriment to islet transplantation. Caspase-3 is the converging point of apoptotic pathways. Therefore, we determined the level of caspase- 3 in the islets transduced with AdvhHGF-hIL-1Ra, following by incubation with the cytokine cocktail of IL- $1 \beta$, TNF- $\alpha$ and IFN$\gamma$. Non-transduced control islets with and without cytokine treatment were used as controls. As shown in Figure 5, caspase-3 activity was high in the un-transducted islets treated with the cytokine cocktail compared to that in the absence of the cytokine cocktail. Transduction of islets with Adv-hHGF-hIL-1Ra protected islets from cytokine-induced apoptosis, as evidence by low increase in caspase- 3 compared to that of non-traduced islets incubated with the cytokines. Since the islets transduced at 2000 MOI showed much higher level of caspase-3 activity compared to that of 1000 MOI transduced islets, we decided to transduce islets at 1000 MOI for in vivo testing.

We also determined the effect of Adv-hHGF-hIL-1Ra transduction on the levels of Bcl-2 (antiapoptotic) and Bax (pro-apoptotic) by Western blot analysis. As shown in Figure 6, there was significant increase in Bcl-2 level, but decrease in Bax level.

\section{Islet Survival and Function after Transplantation}

The effect of hHGF and hIL-1Ra gene expression on the islet survival and function posttransplantation of 1000 islets transduced with Adv-hHGF-hIL-1Ra at 1000 MOI under the kidney capsules of NOD-SCID mice was determined in terms of blood glucose, human insulin, and C-peptide levels. Following transplantation, blood glucose level decreased to around 200 $\mathrm{mg} / \mathrm{dl}$ for both the un-transduced control and Adv-hHGF-hIL-1Ra-transduced islet transplanted mice. However, the mice transplanted with un-transduced islets showed gradual increase in the blood glucose level reaching to $300 \mathrm{mg} / \mathrm{dl}$ at day 28 post-transplantation. In contrast, no increase blood glucose level was seen in case of the mice transplanted with Adv- 
hHGF-hIL-1Ra-transduced islets even at 28 day post-transplantation (Fig. 7). After removal of the kidney containing islets from some mice, blood glucose levels immediately returned to pre-transplant diabetic levels ( $>500 \mathrm{mg} / \mathrm{dL}$ ), confirming that the transplant was responsible for reducing the blood glucose (Fig. 7).

To further define the function of the transplanted islets, glucose tolerance tests were performed at day 27 post-transplantation from the same mice. After intraperitoneal glucose injection, blood glucose values were significantly lower in mice transplanted with Adv-hHGF-hIL-1Ratransduced islets than in mice transplanted with uninfected islets (Figure 8). These results collectively indicate that at 4 weeks after transplantation of an equivalent mass of islets, AdvhHGF-hIL-1Ra-transduced islets lead to superior glucose control and to tolerance as compared with control islets.

Just before the removal of kidney containing islets, blood was collected for measuring the serum insulin and C-peptide levels. As shown in Figure 9A and 9B, serum levels of insulin and c-peptide were significantly higher in mice transplanted with Adv-hHGF-hIL-1Ra transduced islets compared to un-transduced islet transplanted mouse groups, indicating better islet engraftment and function. These results suggest that compared to non-treated islets, coexpression of hHGF and hIL-1Ra sustained the low glucose level of mice for prolonged period, and increased the levels of serum insulin and c-peptide.

\section{Immunohistochemistry}

To determine whether hHGF gene expression after transduction with Adv-hVEGF-hIL-1Ra will promote angiogenesis by human islets, we isolated the kidney bearing Adv-hHGFhIL-1Ra transduced islets at 28 days post-transplantation into diabetic NOD-SCID mice for sectioning and staining with polyclonal rabbit hHGF and von Willebrand Factor (hvWF) antibodies. Staining with anti-human insulin and untransduced islet sections were used as the controls. The positive staining of hHGF was higher in the transduced islets compared to untransduced islets (Fig. 10), suggesting efficient hHGF gene expression after transduction with Adv-hHGF-hIL-1Ra. The positive staining of vWF factor was more evident in Adv-hHGFhIL-1Ra transduced islets, which is an indicator for endothelial cells. Morph metric analysis revealed that Adv-hHGF-hIL-1Ra transduced Islet grafts exhibited higher hHGF and vWFimmunostaining intensity than control islet grafts, with relative intensities of $1.70 \mathrm{vs.} 1$ for hHGF and 1.50 vs. 1 for vWF, respectively. To correlate the degree of islet revascularization with the islet mass in the kidney capsule, we also stained for human insulin. Adv-hHGFhIL-1Ra transduced islets (Fig. 10A) displayed significantly higher levels of insulin content than control islet groups (Fig. 10D), with relative intensity of 1.7 vs. 1. This quantitative difference in insulin content in the kidney capsule between Adv-hHGF-hIL-1Ra transduced and control islet groups of diabetic recipient mice correlated well with their serum insulin and c-peptide profiles (Fig. 9). The positive staining for human insulin also confirmed the functionality of islets. These results indicate the beneficial effect of Adv-hHGF-hIL-1Ra for the islet survival and function post-transplantation.

\section{Discussion}

Isolation and purification of islets are known to disrupt their microvasculature, leading to hypoxia and apoptosis upon transplantation (20). HGF has been shown to increase $\beta$-cell proliferation vitro and ameliorate hyperglycemia in streptozotocin-induced diabetic mice transplanted with murine islet transplantation $(10,11,21)$. Islet injury stimulates resident macrophages and infiltrating leukocytes to produce cytotoxic cytokines like IL-1 $\beta$, TNF- $\alpha$ and IFN- $\gamma$. Among these inflammatory cytokines, IL- $1 \beta$ is the key mediator of $\beta$ cell destruction. Administration of recombinant IL-1Ra protein has been shown to prevent islet dysfunction upon transplantation.(22) Ex vivo transduction of human islets with Adv-hIL-1Ra has been 
reported to prevent IL-1 $\beta$ induced $\beta$ cell impairment and activation of islet cell apoptosis (17). On the other hand, HGF is known to induce angiogenesis, regenerate $\beta$-cells and protect islets from apoptosis. Therefore, the objective of this study was to construct and characterize a bipartite Adv vector, Adv-hHGF-hIL-1Ra, for enhancing the performance of human islet grafts and correction of hyperglycemia upon transplantation under the kidney capsules of STZinduced diabetic NOD-SCID mice.

Apoptosis begins during islet isolation process, peaks 2-3 days post transplantation, and continues for approximately 7 14 days until what remains of the graft has stabilized and become vascularized. Therefore, the use of replication deficient Adv vectors promotes islet engraftment during the first week may be all that is necessary. The lack of integration is an advantage as well, since it will not allow induction of several key cell genes. The viral dose delivered to the patient by ex vivo gene therapy would be small, reducing concerns regarding anaphylaxis. Finally, hHGF and hIL-1Ra co-expression will suppress immune reaction, leading to attenuation of host allergic reactions. Therefore, we decided to use $\Delta \mathrm{E} 1$ and $\Delta \mathrm{E} 3 \mathrm{Adv}$ vector because our goal was to achieve transient gene expression, while avoiding other potential side effects and in vivo virus replication.

Since prolonged incubation with Adv vectors may induce apoptosis and loss of endothelial cells; we decided not to co-culture the islets beyond $12 \mathrm{~h}$, we determined HGF and hIL-1Ra gene expression at protein and mRNA levels. The protein and mRNA levels of HGF and hIL-1Ra increased with increase in MOI (Figs 2 and 3), indicating the Adv vector is indeed expressing these two genes as desired. However, these results cannot be directly correlated, since we used different primers for different genes and thus we can only compare our real time RT-PCR results in relative to the house keeping genes.

Apoptosis is a major pathways through which islets undergo cell death and has been considered as a major factor for primary graft non-function (23). To mimic the in vivo situation of the cytokine mediated insults, we incubated islets with a cocktail of inflammatory cytokines such as IL-1 $\beta$, TNF- $\alpha$ and IFN- $\gamma$. Caspase- 3 is a converging point of apoptosis for intrinsic and extrinsic pathways and caspase- 6 and 7 are generated from caspase-3. There was gradual increase in caspase-3 with increase in Adv-hHGF-hIL-1Ra in the presence of these cytokines, but caspase-3 levels were much lower compared to the un-transduced islets (Fig. 5). The levels of caspase-3 at 500 and 1000 MOI was low, suggesting these Adv vectors are fairly safe at these tested doses.

Bcl-2 family of intracellular proteins plays an important role in regulation of cytochrome $\mathrm{C}$ release into the cytosol and caspase activation. Anti-apoptotic members, such as Bcl-2, Bcl$\mathrm{X}_{\mathrm{L}}$ and Bcl-w are critical for cell survival, whereas pro-apoptotic members, such as Bax, Bad and Bak among others promote apoptosis (24). Heterodimerization of Bcl-2 with Bax or Bak modulates apoptosis, and the ratio of Bcl-2 to Bax or Bak determines survival or death following an apoptotic stimulus. Transduction of islets with Adv-hHGF-hIL-1Ra greatly enhanced the level of Bcl-2 protein while inhibited Bax protein level (Figure 6). This result further demonstrates the protective effect of hHGF and hIL-1Ra co-expression, since Bcl-2 has been shown to inhibit cytochrome $\mathrm{C}$ release and protect against oxidative stress-induced apoptosis (25).

Effect of Adv-hHGF-hIL-1Ra transduction on improvement in the survival and function of islets post-transplantation was determined in streptozotocin-induced diabetic NOD-SCID mice. We observed a reduction in blood glucose levels of mice transplanted with Adv-HGFhIL-1Ra transduced islets than that observed with un-transduced islets, especially after 5 days post-transplantation, but increase in blood glucose levels for the mice transplanted with untransduced islets (Fig. 6). This decrease in blood glucose levels by Adv-hHGF-hIL-1Ra- 
transduced mice correlated with the higher amount of serum insulin and C-peptide secreted by the islets at day 28 post-transplantation (Figures 9A and 9B). Our results are in good agreement with Lopez-Talavera et al.(26), who observed glycemic control in Adv-HGF-transduced islet transplanted rats superior to the tat observed in diabetic control rats.

Revascularization of islet grafts after transplantation is an important process that influences the long-term survival and function of the grafts. Endothelial cells reside within isolated islets and potentially participate in the revascularization process. Immunohistochemical staining in the islet bearing kidney sections at day 28 after transplantation was positive for human insulin, hHGF and vWF (Fig. 10). Positive staining for insulin, which stains pancreatic $\beta$-cells, corroborates the serum insulin data (Fig. 9A) and confirms that islets are indeed functional after transplantation, which is in good agreement with the contemporary literature. vWF is human endothelial cell marker. Therefore, relatively stronger positive staining for hHGF and vWF antigens by the Adv-hHGF-hIL-1Ra transduced islet bearing kidney section groups indicates that blood vessel formation in the transplanted islets was relatively more efficient when islets were transduced with Adv-hHGF-hIL-1Ra prior to transplantation.

These results indicate that HGF and hIL-1Ra co-expression is likely to reduce the islet mass required to achieve normoglycemia. Although we did not examine the apoptosis in graft islet cells, our in vitro data clearly indicated transduction of Adv-HGF-hIL-1Ra led to significant reduction of caspase-3 activity, which is the indicator of apoptosis.

\section{Acknowledgments}

We would like to thank the National Institute of Health (NIH) for financial support (RO1 DK69968).

\section{References}

1. Goss JA, Schock AP, Brunicardi FC, Goodpastor SE, Garber AJ, Soltes G, Barth M, Froud T, Alejandro R, Ricordi C. Achievement of insulin independence in three consecutive type-1 diabetic patients via pancreatic islet transplantation using islets isolated at a remote islet isolation center. Transplantation 2002;74:1761-6. [PubMed: 12499894]

2. Shapiro AM, Lakey JR, Ryan EA, Korbutt GS, Toth E, Warnock GL, Kneteman NM, Rajotte RV. Islet transplantation in seven patients with type 1 diabetes mellitus using a glucocorticoid-free immunosuppressive regimen. N Engl J Med 2000;343:230-8. [PubMed: 10911004]

3. Ryan EA, Lakey JR, Rajotte RV, Korbutt GS, Kin T, Imes S, Rabinovitch A, Elliott JF, Bigam D, Kneteman NM, Warnock GL, Larsen I, Shapiro AM. Clinical outcomes and insulin secretion after islet transplantation with the Edmonton protocol. Diabetes 2001;50:710-9. [PubMed: 11289033]

4. Berneyand T, Ricordi C. Islet cell transplantation: the future? Langenbecks Arch Surg 2000;385:3738. [PubMed: 11127520]

5. Menger MD, Vajkoczy P, Beger C, Messmer K. Orientation of microvascular blood flow in pancreatic islet isografts. J Clin Invest 1994;93:2280-5. [PubMed: 8182162]

6. Narang AS, Cheng K, Henry J, Zhang C, Sabek O, Fraga D, Kotb M, Gaber AO, Mahato RI. Vascular endothelial growth factor gene delivery for revascularization in transplanted human islets. Pharm Res 2004;21:15-25. [PubMed: 14984253]

7. Cheng K, Fraga D, Zhang C, Kotb M, Gaber AO, Guntaka RV, Mahato RI. Adenovirus-based vascular endothelial growth factor gene delivery to human pancreatic islets. Gene Ther 2004;11:1105-16. [PubMed: 15238996]

8. Vajkoczy P, Olofsson AM, Lehr HA, Leiderer R, Hammersen F, Arfors KE, Menger MD. Histogenesis and ultrastructure of pancreatic islet graft microvasculature. Evidence for graft revascularization by endothelial cells of host origin. Am J Pathol 1995;146:1397-405. [PubMed: 7539980]

9. Beattie GM, Montgomery AM, Lopez AD, Hao E, Perez B, Just ML, Lakey JR, Hart ME, Hayek A. A novel approach to increase human islet cell mass while preserving beta-cell function. Diabetes 2002;51:3435-9. [PubMed: 12453897] 
10. Garcia-Ocana A, Vasavada RC, Cebrian A, Reddy V, Takane KK, Lopez-Talavera JC, Stewart AF. Transgenic overexpression of hepatocyte growth factor in the beta-cell markedly improves islet function and islet transplant outcomes in mice. Diabetes 2001;50:2752-62. [PubMed: 11723058]

11. Garcia-Ocana A, Takane KK, Reddy VT, Lopez-Talavera JC, Vasavada RC, Stewart AF. Adenovirusmediated hepatocyte growth factor expression in mouse islets improves pancreatic islet transplant performance and reduces beta cell death. J Biol Chem 2003;278:343-51. [PubMed: 12403787]

12. Benns JM, Choi JS, Mahato RI, Park JS, Kim SW. pH-sensitive cationic polymer gene delivery vehicle: N-Ac-poly(L-histidine)-graft-poly(L-lysine) comb shaped polymer. Bioconjug Chem 2000;11:637-45. [PubMed: 10995206]

13. Roccisana J, Reddy V, Vasavada RC, Gonzalez-Pertusa JA, Magnuson MA, Garcia-Ocana A. Targeted inactivation of hepatocyte growth factor receptor c-met in beta-cells leads to defective insulin secretion and GLUT-2 downregulation without alteration of beta-cell mass. Diabetes 2005;54:2090-102. [PubMed: 15983210]

14. Cardozo AK, Proost P, Gysemans C, Chen MC, Mathieu C, Eizirik DL. IL-1beta and IFN-gamma induce the expression of diverse chemokines and IL-15 in human and rat pancreatic islet cells, and in islets from pre-diabetic NOD mice. Diabetologia 2003;46:255-66. [PubMed: 12627325]

15. Fontaineand MJ, Fan W. Islet cell transplantation as a cure for insulin dependent diabetes: current improvements in preserving islet cell mass and function. Hepatobiliary Pancreat Dis Int 2003;2:1709. [PubMed: 14599964]

16. Tellez N, Montolio M, Biarnes M, Castano E, Soler J, Montanya E. Adenoviral overexpression of interleukin-1 receptor antagonist protein increases beta-cell replication in rat pancreatic islets. Gene Ther 2005;12:120-8. [PubMed: 15578044]

17. Giannoukakis N, Rudert WA, Ghivizzani SC, Gambotto A, Ricordi C, Trucco M, Robbins PD. Adenoviral gene transfer of the interleukin-1 receptor antagonist protein to human islets prevents IL-1beta-induced beta-cell impairment and activation of islet cell apoptosis in vitro. Diabetes 1999;48:1730-6. [PubMed: 10480601]

18. Sandberg JO, Eizirik DL, Sandler S. IL-1 receptor antagonist inhibits recurrence of disease after syngeneic pancreatic islet transplantation to spontaneously diabetic non-obese diabetic (NOD) mice. Clin Exp Immunol 1997;108:314-7. [PubMed: 9158104]

19. Borosand P, Miller CM. Hepatocyte growth factor: a multifunctional cytokine. Lancet 1995;345:2935. [PubMed: 7837864]

20. Narangand AS, Mahato RI. Biological and biomaterial approaches for improved islet transplantation. Pharmacol Rev 2006;58:194-243. [PubMed: 16714486]

21. Nakano M, Yasunami Y, Maki T, Kodama S, Ikehara Y, Nakamura T, Tanaka M, Ikeda S. Hepatocyte growth factor is essential for amelioration of hyperglycemia in streptozotocin-induced diabetic mice receiving a marginal mass of intrahepatic islet grafts. Transplantation 2000;69:214-21. [PubMed: 10670629]

22. Sandberg JO, Eizirik DL, Sandler S, Tracey DE, Andersson A. Treatment with an interleukin-1 receptor antagonist protein prolongs mouse islet allograft survival. Diabetes 1993;42:1845-51. [PubMed: 8243831]

23. Ramachandran S, Desai NM, Goers TA, Benshoff N, Olack B, Shenoy S, Jendrisak MD, Chapman WC, Mohanakumar T. Improved islet yields from pancreas preserved in perflurocarbon is via inhibition of apoptosis mediated by mitochondrial pathway. Am J Transplant 2006;6:1696-703. [PubMed: 16827873]

24. Hanke J. Apoptosis and occurrence of Bcl-2, Bak, Bax, Fas and FasL in the developing and adult rat endocrine pancreas. Anat Embryol (Berl) 2000;202:303-12. [PubMed: 11000281]

25. Jiangand X, Wang X. Cytochrome C-mediated apoptosis. Annu Rev Biochem 2004;73:87-106. [PubMed: 15189137]

26. Lopez-Talavera JC, Garcia-Ocana A, Sipula I, Takane KK, Cozar-Castellano I, Stewart AF. Hepatocyte growth factor gene therapy for pancreatic islets in diabetes: reducing the minimal islet transplant mass required in a glucocorticoid-free rat model of allogeneic portal vein islet transplantation. Endocrinology 2004;145:467-74. [PubMed: 14551233] 

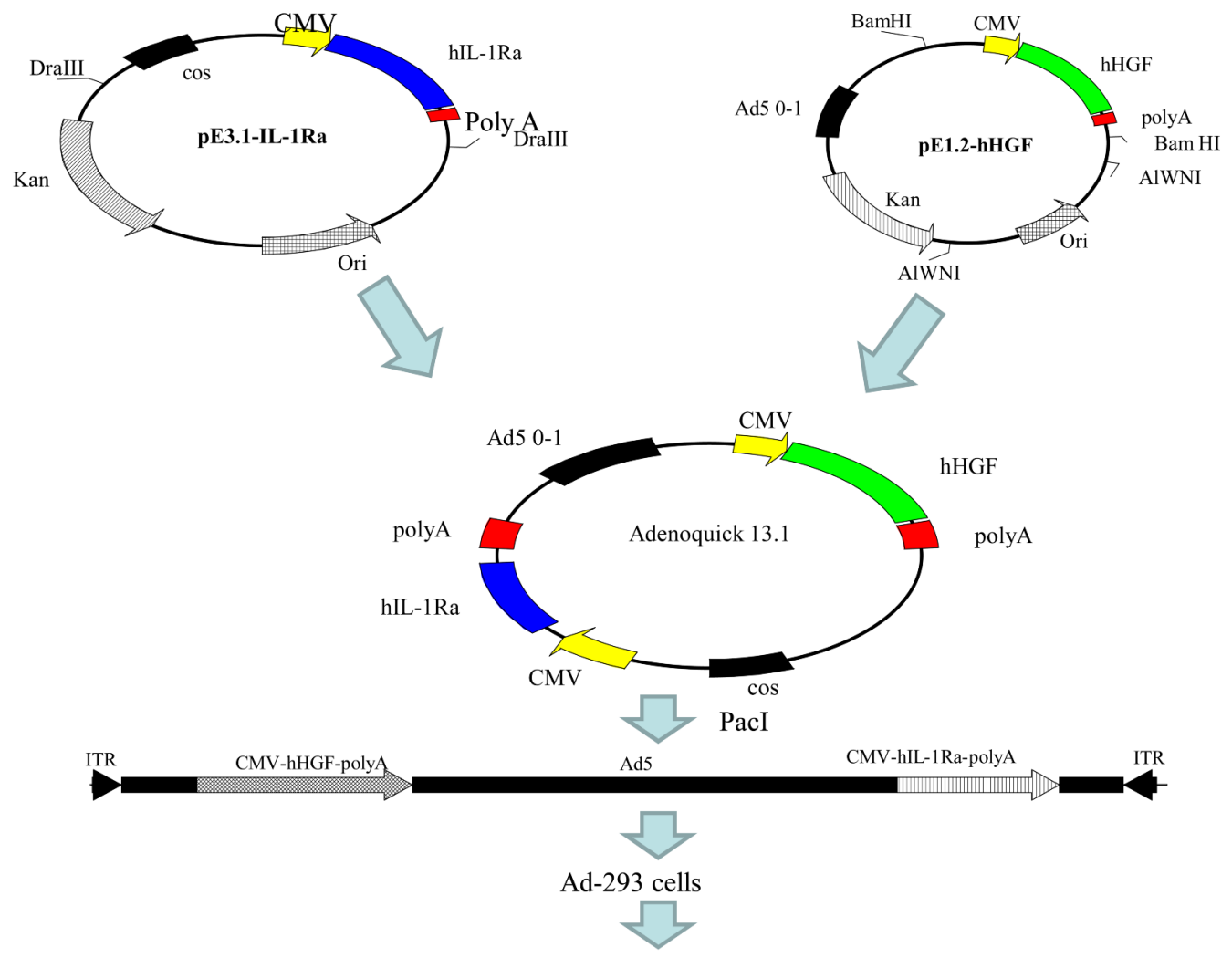

Adv-hHGF-hIL-1Ra

Figure 1.

Construction of E1 and E3 deleted bipartite adenoviral (Adv) vector encoding hHGF and hIL-1Ra using AdenoQuick cloning system. Adv-HGF-hIL-1Ra was constructed by excising hHGF and hIL-1Ra form vector pUMVC and cloning into the multiple cloning sites of shuttle plasmids pE 3.1 and pE 1.2 using NotI. The expression cassettes of hHGF and hIL-1Ra containing the CMV promoter were cloned into AdenoQuick plasmid 13.1 to generate a cosmid containing the entire sequence of recombinant adenovirus. After transfection into 293 cells, recombinant adenovirus Adv-hHGF-hIL-1Ra was produced. 

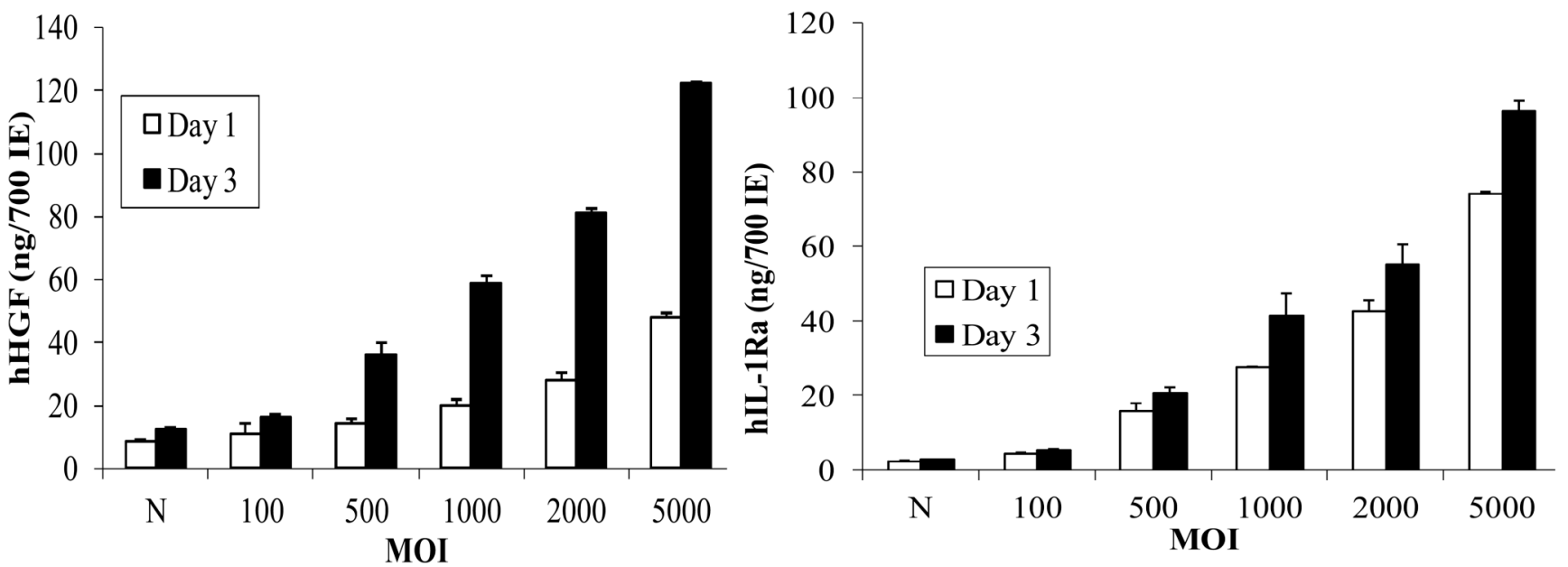

Figure 2.

Time profile of hHGF and hIL-1Ra gene expression at protein levels in human islets transduced Adv-HGF-IL-1Ra at different multiplicities of transduction (MOI) 100, 500, 1000, 2000 and 5000 per 1000 islets. Non-transduced islets were used as controls. ELISA of cell culture supernatants were performed at days 1 and 3. Cumulative expression levels were presented as the mean \pm S.D. $(n=4)$. N, non-transduced control islets. 

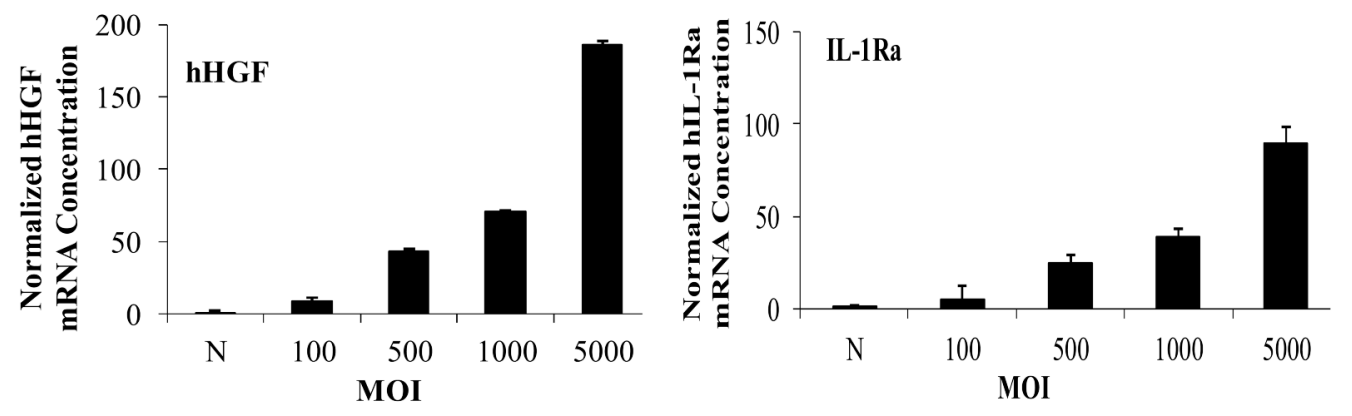

Figure 3.

Real-time PCR of human islets at day 3 post transduction with AdvHGF-IL-1Ra at different MOI of 100, 500, 1000, 2000 and 5000. Forward and reverse primers were designed from hIL-1Ra and HGF genes. Non-transduced islets were used as controls. Cell pellet was collected at day 3 and processed for mRNA extraction, reverse transcription and real time PCR using SYBR green chemistry. The normalized mRNA values of the different MOI treated islets are plotted. Since different primers were used, no direct comparison can be made, other than demonstration of increase in mRNA levels with increase in MOI. N, non-transduced control islets. 


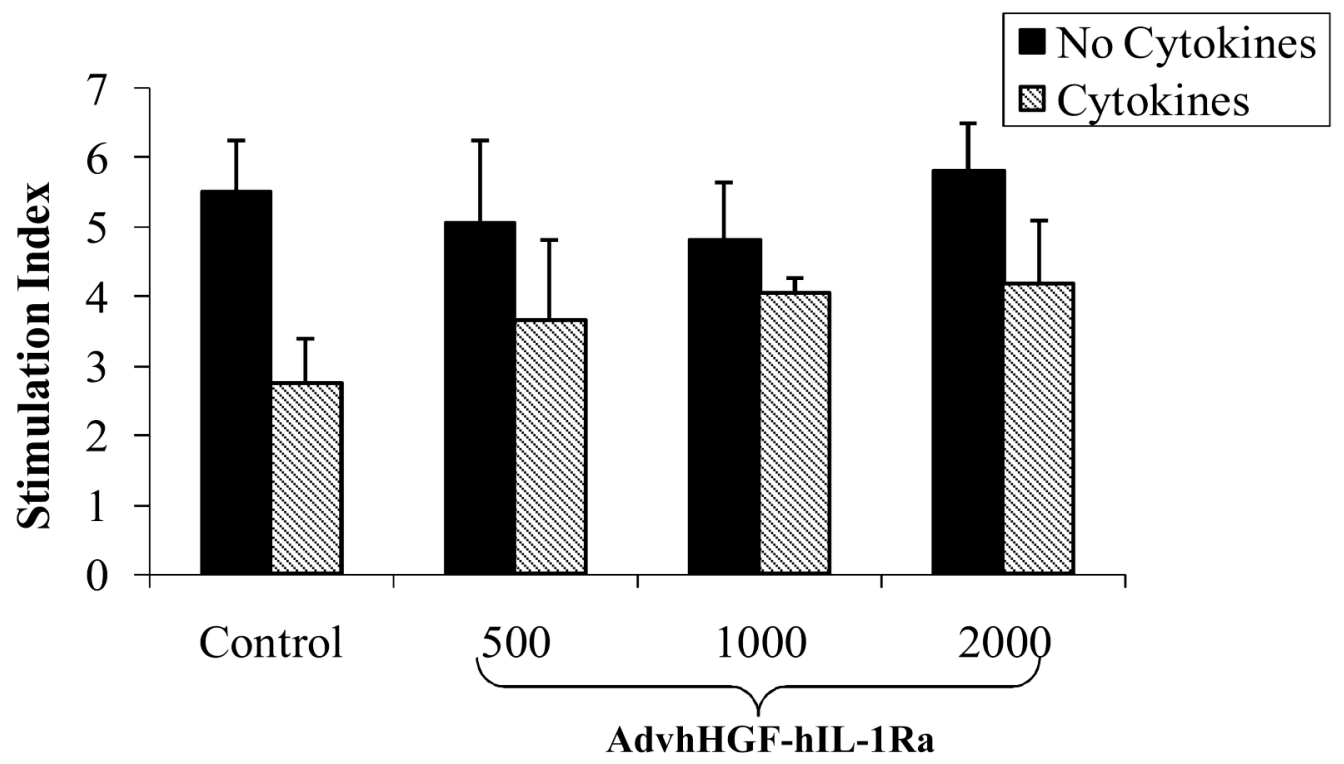

Figure 4.

Stimulation index (SI) of human islets with or without cytokine incubation after transduction with Adv-hHGF-IL-1Ra at 500, 1000 and 2000 MOI. Non-transduced islets were used as controls. SI was determined as the ratio of insulin release from islets when they are incubated at $396 \mathrm{mg} / \mathrm{dl}$ and $45 \mathrm{mg} / \mathrm{dl}$ glucose containing media. Increase in insulin secretion in response to high glucose concentration and decrease with reducing concentration is significant of metabolically healthy and functional islets. Results are expressed as a mean \pm S.D of $n=4$. 


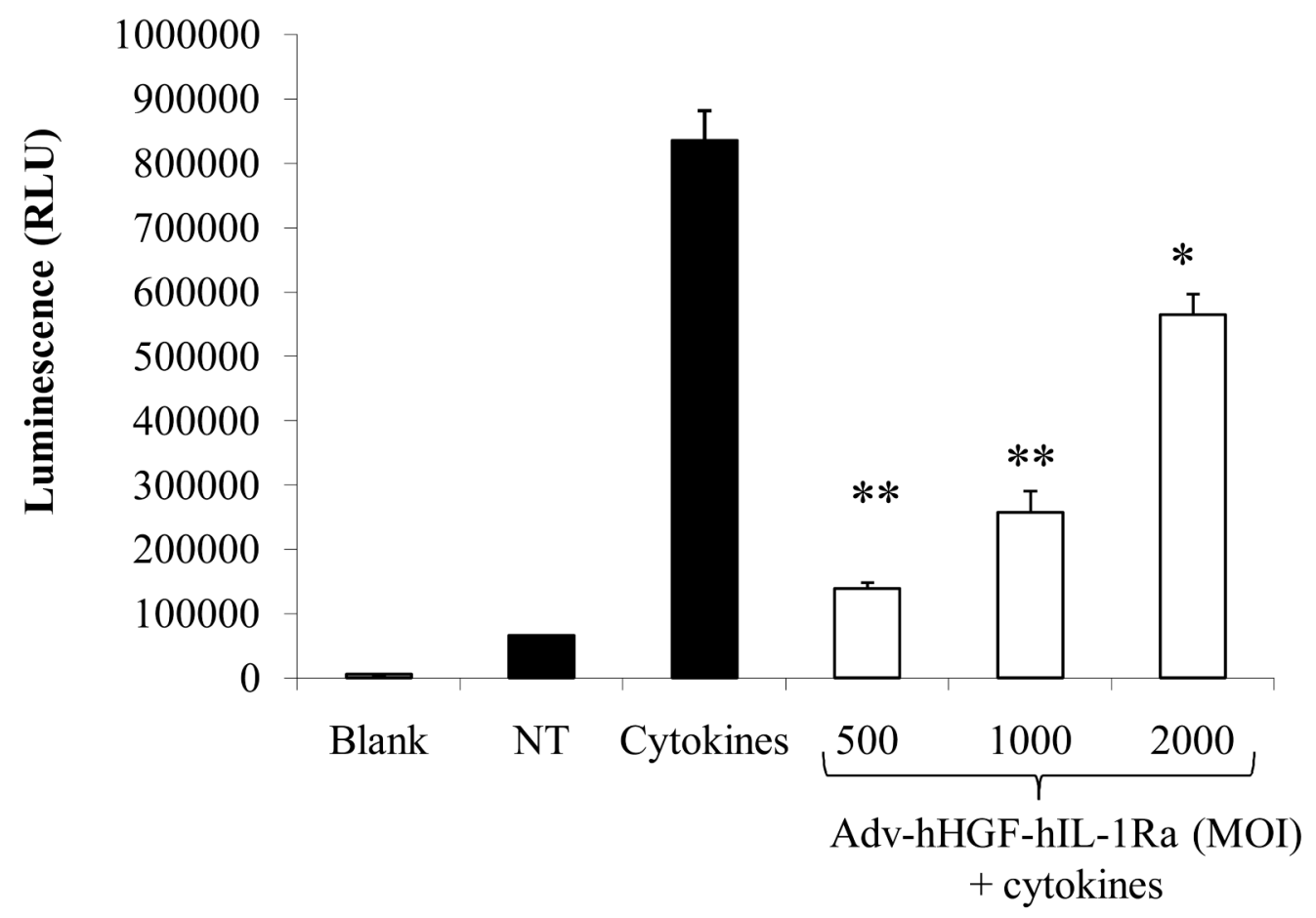

Figure 5.

Effect of hHGF and hIL-1Ra co-expression on caspase-3 activity. Following transduction with Adv-hHGF-hIlL-1Ra, islets were incubated with a cocktail of cytokines, such as IL- 1 $\beta$ (10ng/ $\mathrm{ml}), \mathrm{TNF}-\alpha(10 \mathrm{ng} / \mathrm{ml})$ and INF- $\gamma(50 \mathrm{ng} / \mathrm{ml})$. Non-transduced islets with and without cytokine treatment were used as controls. Blank media was also used as negative controls. Caspase-3 activity was determined at day 3 post-transduction by measuring the amount of caspase- 3 present in the lysate of islets after cell lysis using a Caspase-Glo 3/7 luciferase assay. Results are expressed as the mean \pm S.D. of $n=4$. $* \mathrm{p}<0.05$ and $* * \mathrm{p}<0.02$, compared to non-transduced islets with cytokine treatment. NT, non-transduced islets without cytokine treatment. 


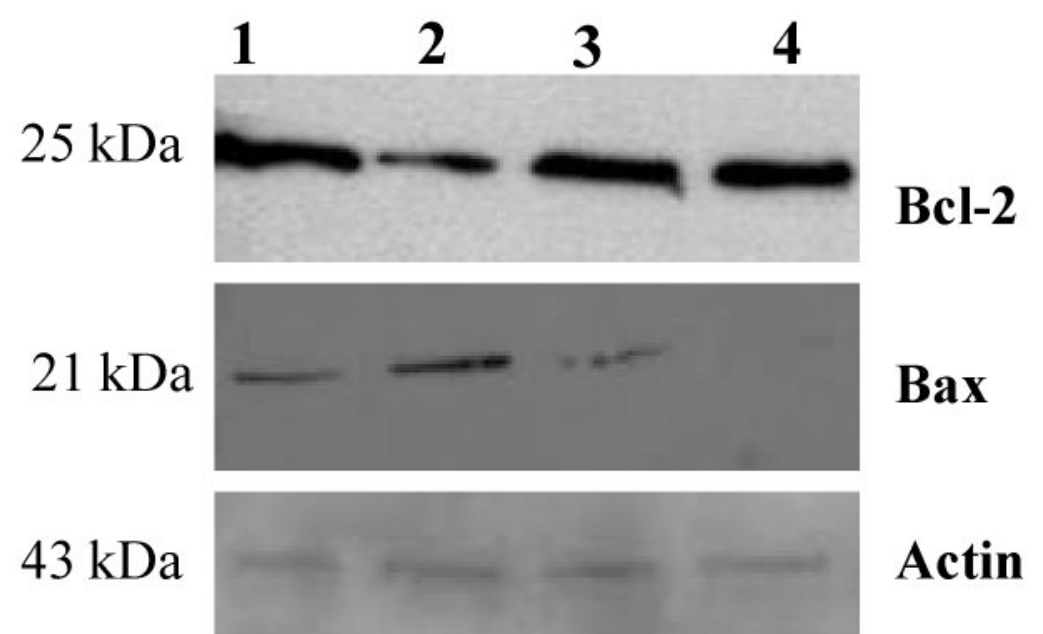

Figure 6.

Effect of hHGF and hIL-1Ra co-expression on Bcl-2 and Bax protein levels. Following transduction with Adv-hHGF-hIlL-1Ra, islets were incubated with a cocktail of cytokines, such as IL- $1 \beta(10 \mathrm{ng} / \mathrm{ml})$, TNF- $\alpha(10 \mathrm{ng} / \mathrm{ml})$ and INF- $\gamma(50 \mathrm{ng} / \mathrm{ml})$. Non-transduced islets with and without cytokine treatment were used as controls. Bcl-2 and Bax was determined at day 3 post-transduction by Western blot analysis. Lane 1, non-transduced islets; lane 2, nontransduced islets with cytokine treatment; lane 3, islets transduced with Adv-hHGF-hIL-1Ra at $500 \mathrm{MOI}$ with cytokine treatment; and lane 4, islets transduced with Adv-hHGF-hIL-1Ra at $1000 \mathrm{MOI}$ with cytokine treatment. 


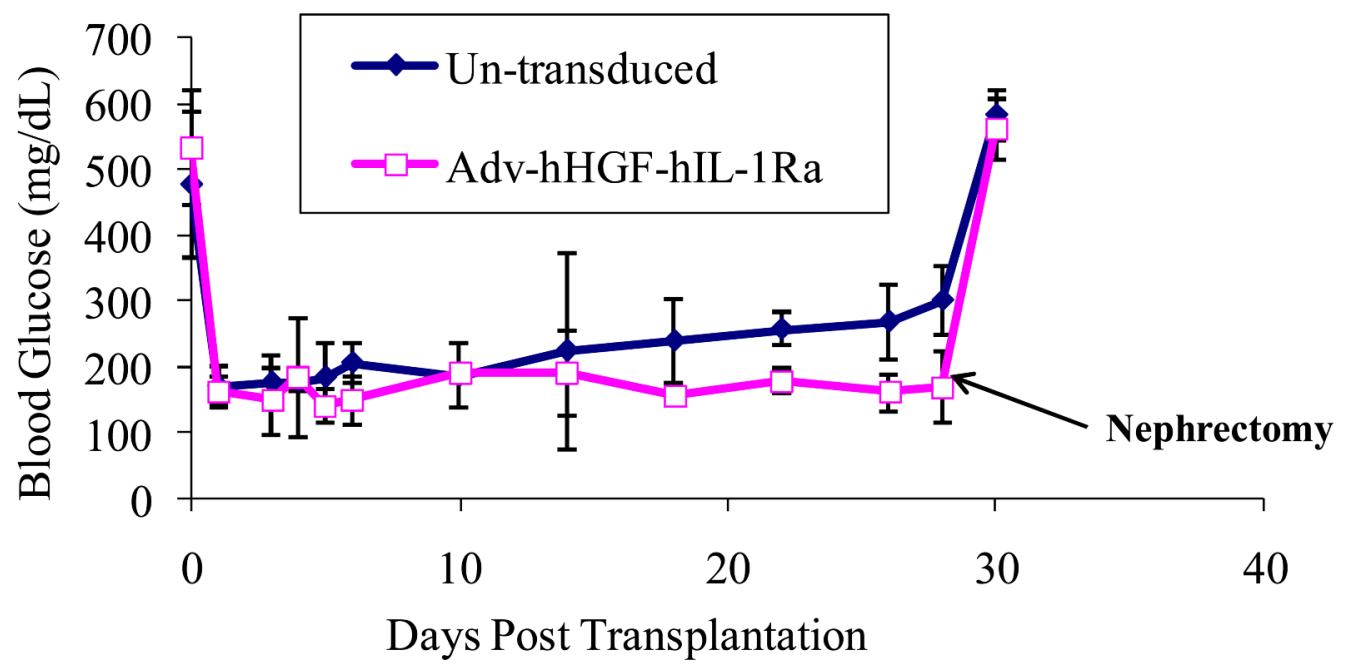

Figure 7.

Effect of Adv-hHGF-hIL-1Ra transduction on the outcome of islet transplantation. Following transduction with Adv-hHGF-hIL-1Ra at 1000 MOI, 1000 islets were transplanted under the kidney capsule of streptozotocin-induced diabetic NOD-SCID mice. The mice transplanted with un-transduced islets were used as controls. The nonfasting glucose levels were measured in each animal up to 28 days post-transplantation using a One Touch Ultra Glucometer (LifeScan, Inc.). 


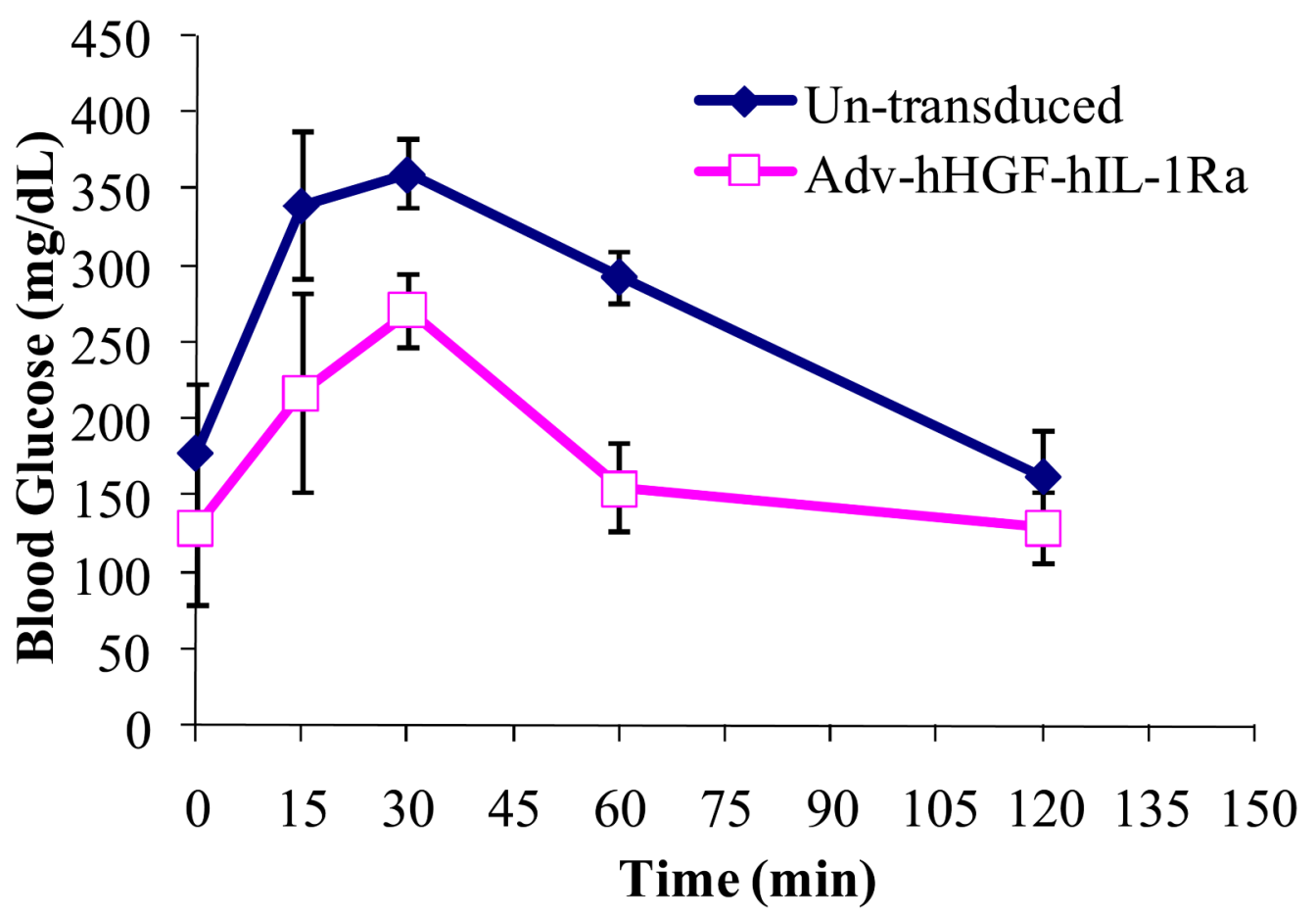

Figure 8.

Glucose tolerance in streptozotocin-induced NOD-SCID mice transplanted with uninfected or Adv-hHGF-hIL-1Ra transduced islets. At day 27 after the transplant, mice were fasted overnight and then injected intraperitoneally with glucose $(2 \mathrm{~g} / \mathrm{kg}$ of body weight). Blood glucose levels were measured from the snipped tail at the time points indicated with a glucometer. Results are the mean \pm S.D. of $n=4$. $* P<0.05$ compared with control using ANOVA. 

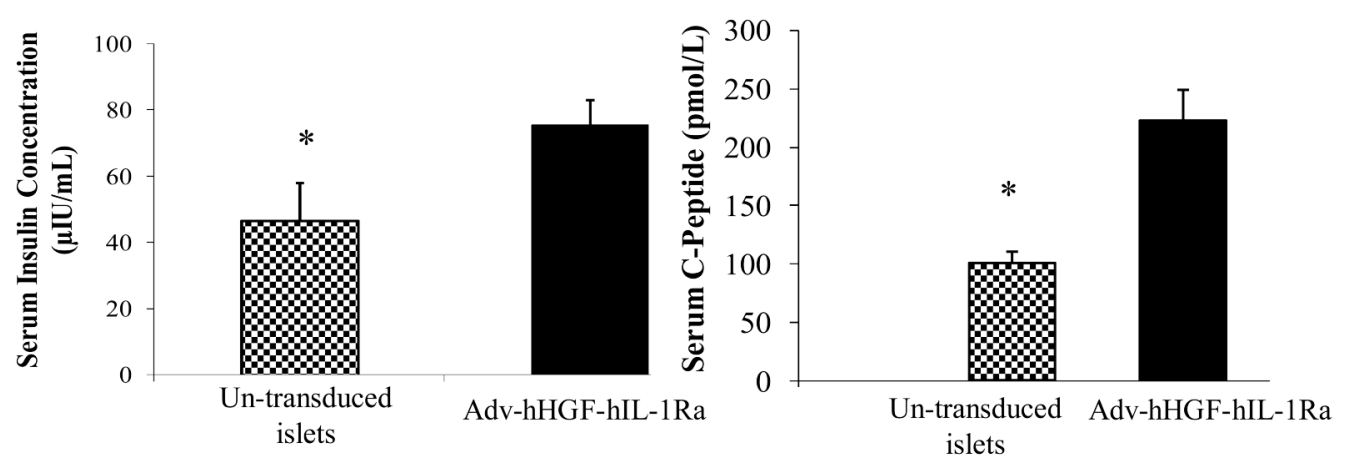

Figure 9.

Effect of Adv-hHGF-hIL-1Ra transduction on serum insulin and c-peptide levels. Following ex vivo transduction with Adv-hHGF-hIL-1Ra at 1000 MOI, 1000 islets were transplanted under the kidney capsule of streptozotocin-induced diabetic NOD-SCID mice. The mice transplanted with un-transduced islets were used as controls. At day 28 post-transplantation, the mice were sacrificed and blood was collected to measure serum insulin and C-peptide by ELISA. Results are expressed as the mean \pm S.D. $(n=4) .{ }^{*} p<0.05$. 


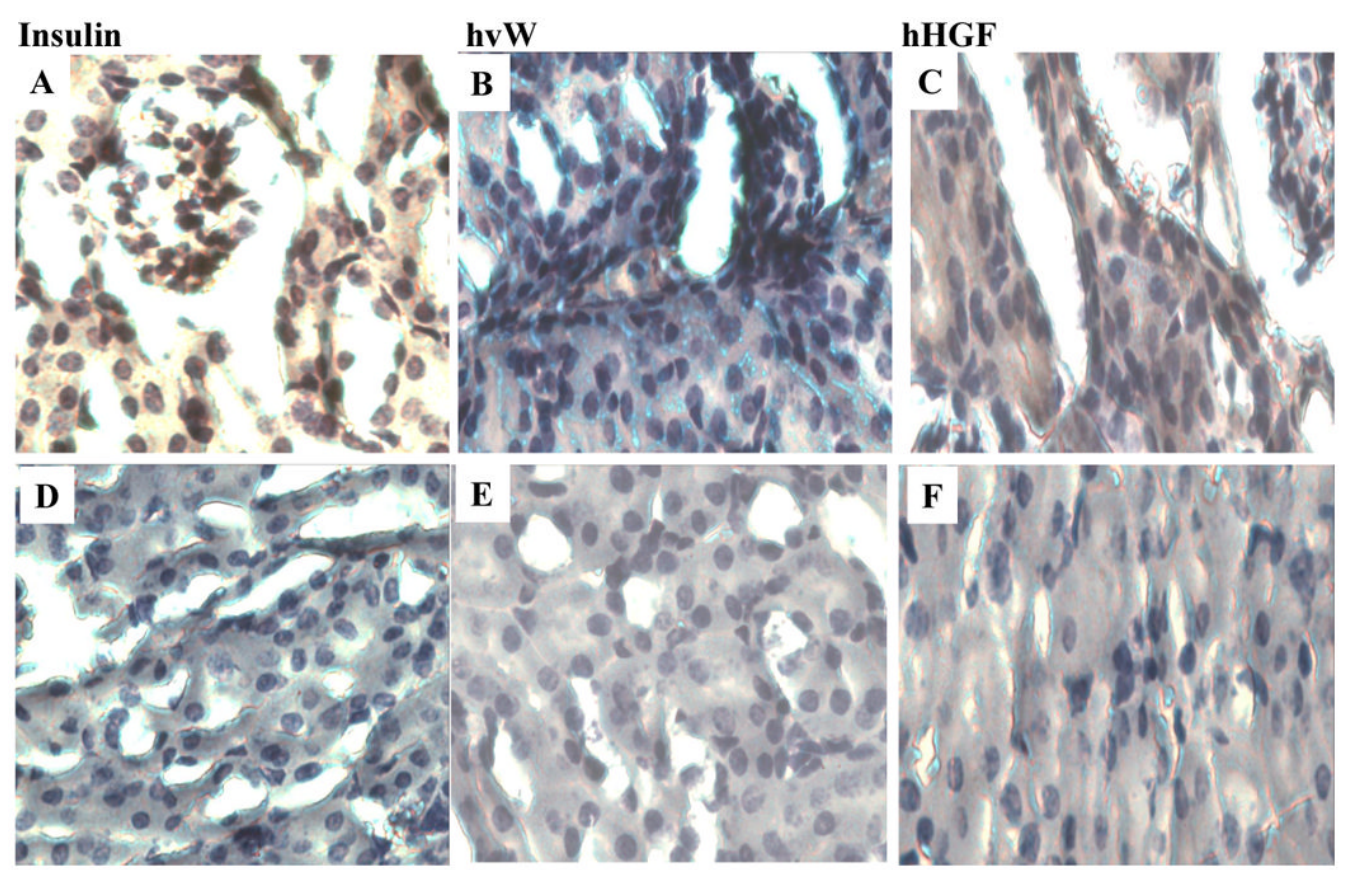

Figure 10.

Immunohistochemical staining of islet bearing kidney sections at 28 days post-transplantation of human islets transduced with Adv-hHGF-hIL-1Ra at 1000 MOI. Rabbit polyclonal antihuman HGF and hvW were used for detecting hHGF and endothelial cells. A) insulin, B) hvW and C) hHGF staining for Adv-hHGF-hIL-1Ra-transduced islets; and D) insulin, E) hvW and F) hHGF staining for the control islets. 